\title{
ON GLOBAL CONVERGENCE OF FORCED NONLINEAR DELAY DIFFERENTIAL EQUATIONS AND APPLICATIONS
}

\author{
D. D. HAI AND C. QIAN
}

Abstract. Consider the following nonlinear delay differential equation with a forcing term $r(t)$ :

$$
x^{\prime}(t)+a(t) x(t)+b(t) f(x(t-\tau(t)))=r(t), t \geqslant 0,
$$

where $a \in C[[0, \infty),[0, \infty)], b, \tau \in C[[0, \infty),(0, \infty)], r \in C[[0, \infty), \mathbb{R}], f \in C[(L, \infty),(L, \infty)]$ with $-\infty \leqslant L \leqslant 0$, and $\lim _{t \rightarrow \infty}(t-\tau(t))=\infty$. We establish a sufficient condition for every solution of the equation to converge to zero. By applying the result to some special cases and differential equation models from applications, we obtain several new criteria on the global convergence of solutions.

Mathematics subject classification (2010): 34K20.

Keywords and phrases: nonlinear delay differential equations, forcing term, fixed points, global convergence, biological models.

\section{REFERENCES}

[1] L. BerezAnSKy AND E. BRAVERMAn, New stability conditions for linear differential equations with several delays, Abstr. Appl. Anal., 2011 (2011) (Article ID 178568) 19p.

[2] L. BereZANS KY, E. BRAVERMAN AND L. IDEls, Mackey-Glass model of hematopoiesis with monotone feedback revisited, Appl. Math. Comput., 219 (2013), 4892-4907.

[3] L. Berezansky AND E. Braverman, Stability conditions for scalar delay differential equations with a non-delay term, Appl. Math. Comput., 250 (2015), 157-164.

[4] El-Morshedy, Global attractivity in a population model with nonlinear death rate and distributed delays, J. Math. Anal. Appl., 410 (2014), 642-658.

[5] J. R. GRAEF AND C. QIAN, Global attractivity in differential equations with variable delays, J. Austral. Math. Soc. Ser. B, 41 (2000), 568-579.

[6] D. D. HAI AND C. QIAN, Global attractivity in nonlinear difference equations of higher order with a forcing term, Appl. Math. Comput., 264 (2015), 198-207.

[7] K. Gopalsamy, M. R. S. Kulenovic and G. Ladas, Time lags in a "food limited" population model, Appl. Anal., 31 (1988), 225-237.

[8] K. Gopals amy, Stability and Oscillations in Delay Differential Equations of Population Dynamics, Mathematics and its Applications, 74, Kluwer Academic Pulishers Group, Dordrecht, 1992.

[9] I. GyORI AND G. LADAS, Oscillation Theory of Delay Differential Equations with Applications, The Clarendon Press, Oxford Science Publications, NY, 1991.

[10] Y. KuAng, Delay Differential Equations with Applications in Population Dynamics, Mathematics in Science and Engineering, 191, Academic Press, Boston, 1993.

[11] BEG-BIN LIM, Asymptotic bounds of solution of functional differential equation $x^{\prime}(t)=a x(\lambda t)+$ $b x(t)+f(t), 0<\lambda<1$, SIAM J. Math. Anal., 9 (1978), 915-920.

[12] E. LiZ AND G. Rost, Dichotomy results for delay differential equations with negative Schwarzian derivative, Nonlinear Anal., 11 (2010), 1422-1430.

[13] M. C. Mackey, L. Glass, Oscillation and chaos in physiological control systems, Science, 197 (1977), 287-289.

[14] C. QIAN, Global attractivity of solutions of nonlinear delay differential equations, Dynam. Contin. Discrete Impuls. Systems, 13B (2006), 25-37. 
[15] C. QIAN, Global attractivity in a nonlinear delay differential equation with applications, Nonlinear Anal., 71 (2009), 1893-1900.

[16] C. QIAN, Global attractivity in a variable coefficient nonlinear delay differential equation, Comm. Appl. Nonlinear Anal., 20 (2013), 33-44.

[17] C. QIAN AND Y. SUN, Global attractivity of solutions of nonlinear delay differential equations with a forcing term, Nonlinear Anal., 66 (2007), 689-703.

[18] M. WAZEws Ka-CZyZewska And A. Lasota, Mathematical problems of the dynamics of the redblood cells system, Ann. Polish Math. Soc. Series III, Appl. Math., 17 (1988), 23-40. 